(2) Open Access Full Text Article

\title{
Atypical glomus tumor of uncertain malignant potential in the urinary bladder
}

\author{
This article was published in the following Dove Press journal: \\ Pathology and Laboratory Medicine International \\ 9 December 2010 \\ Number of times this article has been viewed
}

\author{
Lindsay L Waters' \\ Qihui “Jim” Zhai \\ J Scott Buie ${ }^{2}$ \\ Rodney Miller ${ }^{2}$ \\ John A Pumphrey \\ Sang $\mathrm{Wu}^{2}$
}

'Department of Pathology, The Methodist Hospital, Houston, TX, USA; ${ }^{2}$ ProPath Associates, Dallas, TX, USA; ${ }^{3}$ Associates of Urology, Fort Worth, TX, USA; ${ }^{4}$ University of Cincinnati and Greater Cincinnati Pathologists Inc, Cincinnati, OH, USA

\begin{abstract}
We present a case of an atypical glomus tumor of uncertain malignant potential in an 84-year-old woman with accompanied recurrent low-grade papillary urothelial carcinoma of the urinary bladder. Glomus tumor frequently occurs in the dermis or subcutis of extremities; its morphology includes round cells with pale-eosinophilic cytoplasm and central punched-out round to oval nucleus. Immunostains demonstrate muscle-specific- actin and desmin positivity and CD 34 negativity. Atypical glomus tumors of uncertain malignant potential are defined by lack of frank malignant features, in addition to high mitotic count, superficial location, or large size only, or deep location only. Our case is characterized by an abnormal proliferation of ovoid cells in the lamina propria, uniform ovoid nuclei with indistinct eosinophilic cytoplasm, sheet-like growth pattern with a prominent capillary network, mild nuclear atypia, and high mitotic rate. Immunostains showed tumor cells are positive for smooth muscle actin, smooth muscle myosin, and negative for CD 34. An English literature search revealed only one case of malignant glomus tumor but no atypical glomus tumors occurring in the urinary bladder. We described a case of atypical glomus tumor of uncertain malignant potential occurring in an unusual location, the urinary bladder.
\end{abstract}

Keywords: atypical glomus tumor, glomus tumor of uncertain malignant potential, urothelial carcinoma, urinary bladder

\section{The case}

An 84-year-old woman presented with a history of low grade superficial urothelial carcinoma of the bladder, first resected in June 2005. Follow-up revealed recurrences at the right posterolateral bladder wall, which was treated with an indigo laser in January 2006 and a repeat resection in June 2006. Next, routine follow-up cystoscopy showed a small recurrence at the left dome of the bladder. Transurethral resection of the bladder tumor was performed and multiple fragments of irregular pink-tan tissue $(1.5 \times 1.1 \times 0.6 \mathrm{~cm})$ were removed.

\section{Discussion of diagnosis}

Surgically resected tissue was fixed in $10 \%$ neutral formalin, sections of the specimen were stained with hematoxylin and eosin (H\&E), and immunohistochemical staining of the tissue was performed.

Histological examination reveals recurrent low grade papillary urothelial carcinoma (Figure 1). There is a separate lesion incidentally identified in the laminar propria, measuring $3.0 \mathrm{~mm}$ in greatest dimension. Microscopically (Figure 2), this lesion is characterized by an abnormal proliferation of ovoid cells in the lamina propria, uniform
Correspondence: Qihui “Jim” Zhai University of Cincinnati, Department of Pathology, 23I Albert Sabin Way-Room I358, ML 0529,

Cincinnati, OH, 45267-0529, USA

$\mathrm{Tel}+\mathrm{I} 5135843838$

Email qihui.zhai@uc.edu 


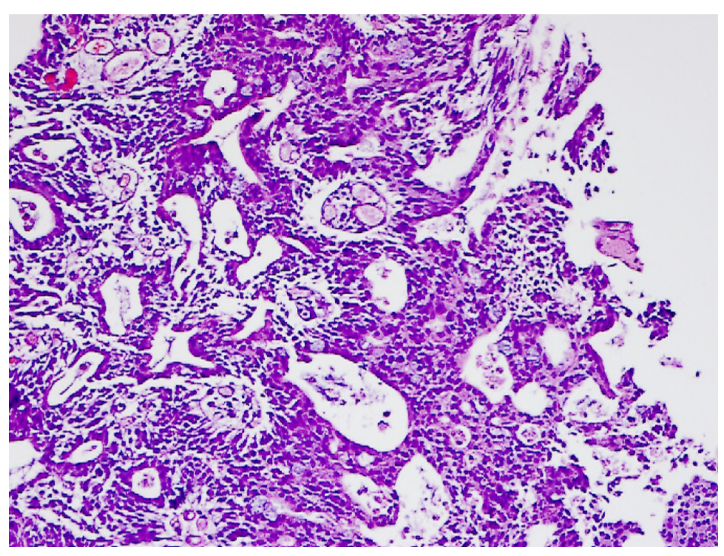

Figure I H\&E histology of the superficial low grade papillary urothelial carcinoma (I0X).

ovoid nuclei with indistinct eosinophilic cytoplasm, sheet-like growth pattern with a prominent capillary network, mild nuclear atypia, and high mitotic rate (2/HPF). Mitotic activity is not difficult to identify, and some individual high power fields contain 4 or 5 mitotic figures. The small size of the lesion precludes a count of 50 high power fields, although there were 23 mitotic figures in 10 high power fields, which extrapolates to 115 mitosis per 50 high power fields. No atypical mitotic figures, cytologic atypia, or necrotic areas are seen. However, occasional individually chronic (apoptotic) tumor cells are noted in the background.

Glomus tumors are mesenchymal neoplasms comprising cells closely resembling smooth muscle cells of the normal glomus body. ${ }^{1,2}$ These uncommon neoplasms account for less than $2 \%$ of soft tissue tumors. ${ }^{2,3}$ Glomus tumors are characterized by small size $(<1 \mathrm{~cm})$, benign nature, and location often in the dermis or subcutis of extremities. Occasional glomus tumors may exhibit unusual clinical features, such as large size, deep soft tissue or visceral location, infiltrative growth pattern, or multicentricity. ${ }^{4}$ Histological features of glomus tumors include uniformly round cells with pale eosinophilic cytoplasm and central punched-out round to oval nucleus. Atypical glomus tumors are exceedingly rare. Histologically, they are defined by atypical features and lack of criteria for malignant glomus tumor. Typically, glomus tumors are immunohistochemically positive for smooth muscle actin and myosin and negative for CD 34 .

Immunostains show that the tumor cells are positive for smooth muscle actin and smooth muscle myosin (Figure 3). Other markers, including cytokeratin LMW $(8,18)$, cytokeratin
A

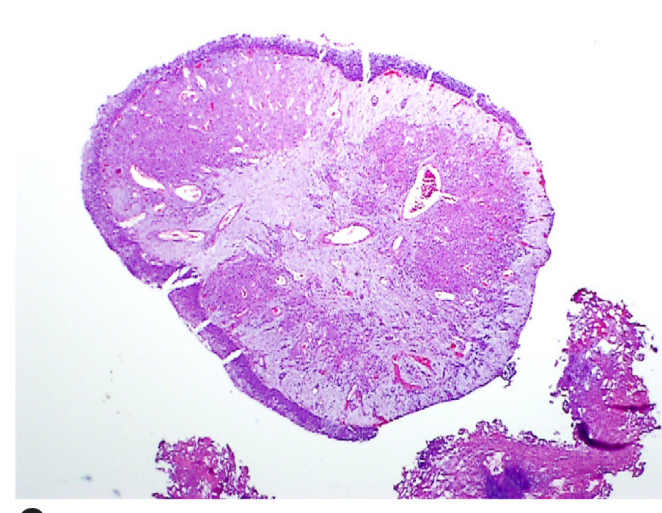

C

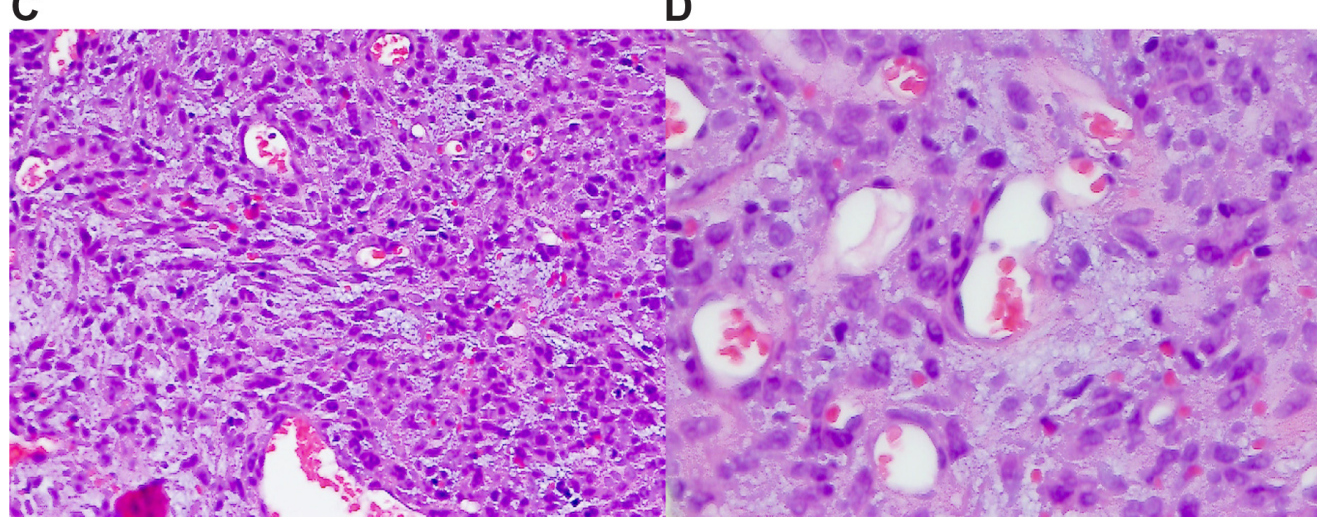

B

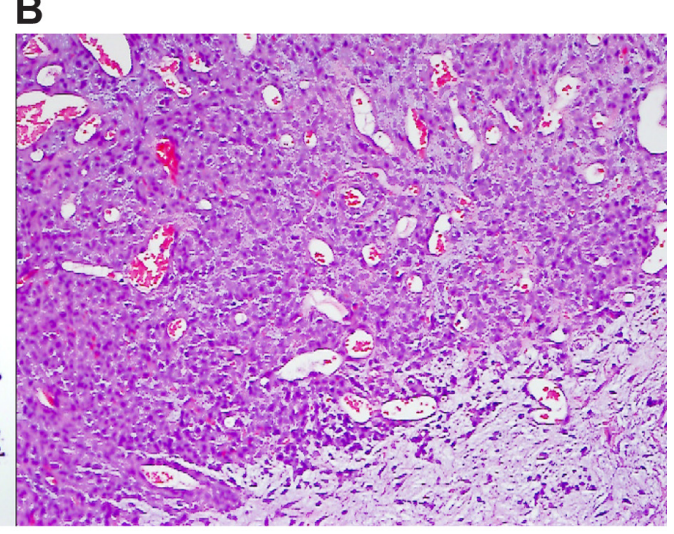

D

$$
\text { (1) }
$$




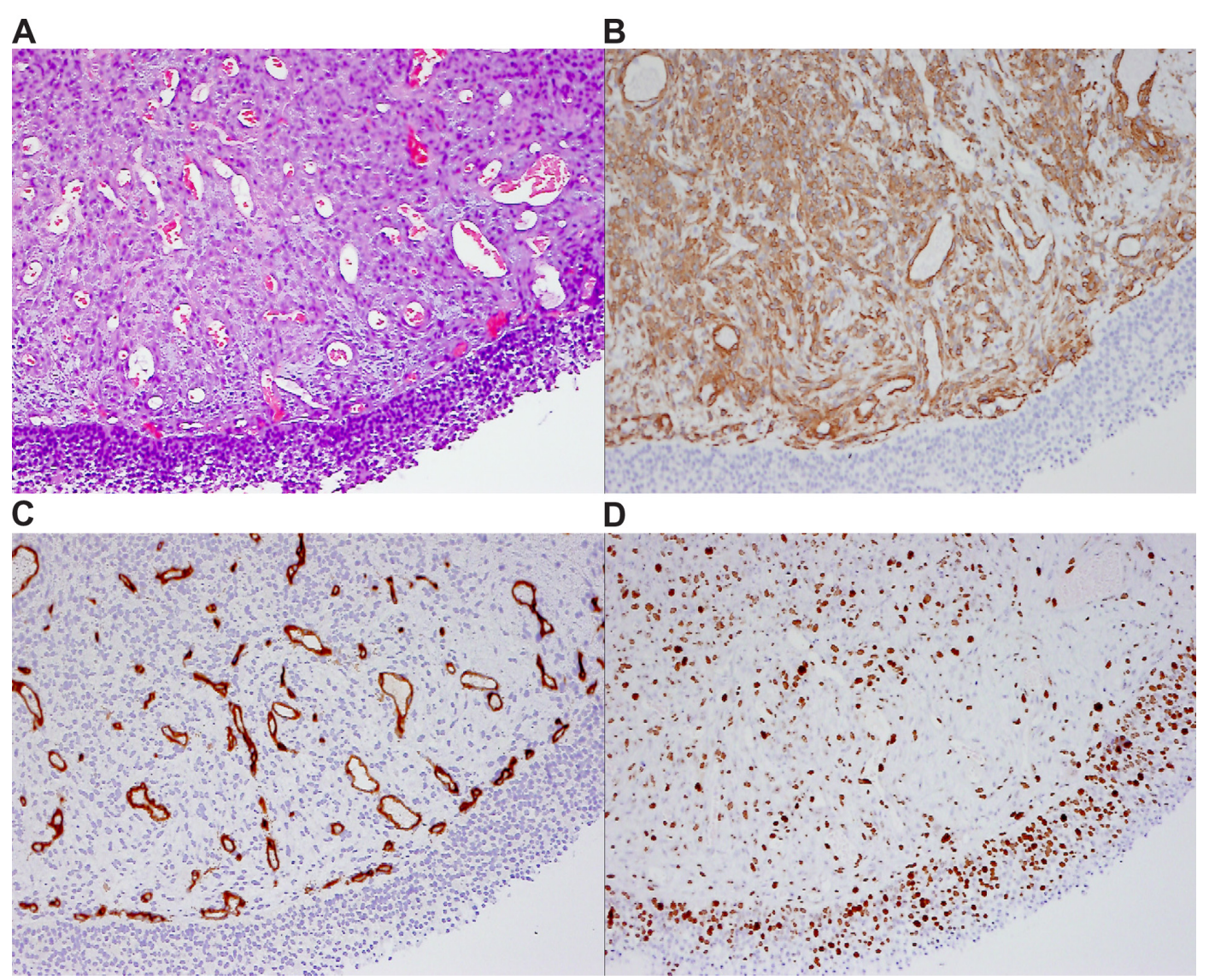

Figure 3 Immunoprofile for atypical glomus tumor of uncertain malignant potential. A) I0X power H\&E photo; B) Smooth muscle actin (I0X) showing tumor cells are positive; C) CD34 (I0X) immunostain showing the capillary endothelial cells are positive, but the tumor cells are negative; D) Nuclear staining of Ki-67 (I0x) showing significant nuclear positivity.

HMW (34ßE12), chromogranin, synaptophysin, S-100 protein, MART-1, CD34, CH117 (c-kit), CD31, WT1, SOX10, desmin, CD57, and estrogen receptor immunostains are negative. High Ki-67 proliferative index, an estimated $40 \%$ (Figure 4), indicates that this is not a regular benign glomus tumor. The constellation of H\&E histological features and

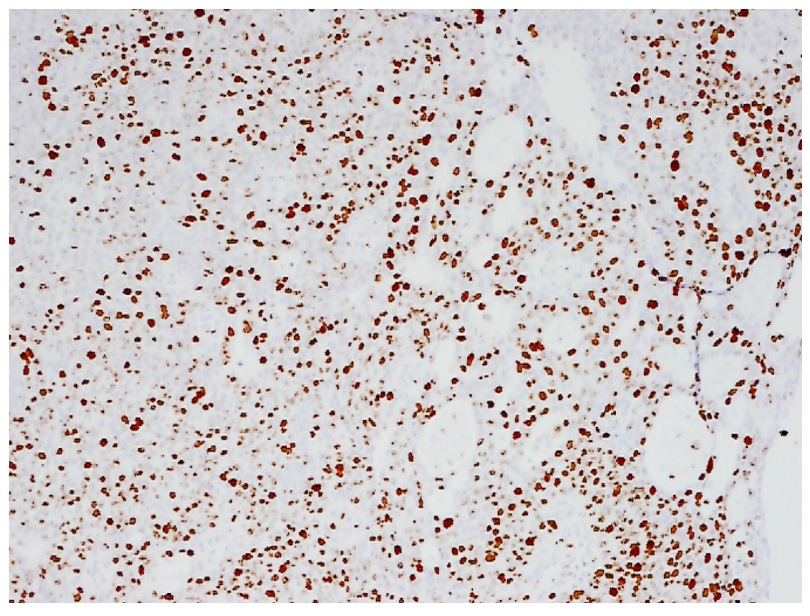

Figure 4 Ki-67 immunostaining (10x) showing a brisk proliferative activity with an estimated $40 \%$ of the tumor cells are positive in nuclear pattern. immunoprofile supports the diagnosis of an atypical glomus tumor of uncertain malignant potential.

\section{Treatment and management}

Glomus tumors are generally benign and solitary. This unique case of atypical glomus tumor of uncertain malignant potential represents a challenging, rare neoplasm occurring in an unusual location that encourages review of diagnostic criteria for atypical glomus tumors. The classification and characterization of glomus tumors was proposed by Folpe et al. ${ }^{1}$ All types of glomus tumors typically have the immunohistochemical profile of smooth muscle actin positivity, while desmin, CD 34, cytokeratin, and S-100 are typically negative. ${ }^{3}$ Specifically, atypical glomus tumors of uncertain malignant potential are defined by lack of criteria for glomangiosarcoma, in addition to the following: 1) high mitotic count ( $>5 / 50 \mathrm{HPF}$ ) and superficial location; 2) large size only; or 3) deep location only. ${ }^{1,3}$

Histological and immunohistochemical examination aid in differentiating this tumor from others which may generate diagnostic challenges, such as benign glomus tumor, glomangiosarcoma, hemangiopericytoma, and epitheloid 
leiomyosarcoma. Benign glomus tumors are characterized by small, uniform, rounded cells with indistinct nucleoli. ${ }^{1-3}$

Glomangiosarcomas are characterized by: 1) size $>2 \mathrm{~cm}$ and deep location; 2) atypical mitotic figures; or 3) moderate to high nuclear grade and 5 or more mitotic figures per 50 high power fields. ${ }^{5}$ Hemangiopericytoma is another entity that may resemble glomus tumors. However, they have staghorn-type vessels, spindle cells, and elongated nuclei, and immunohistochemical staining pattern of smooth muscle actin negativity and CD 34 positivity., ${ }^{1,2,6-8}$ Epitheloid leiomyosarcomas are typically identified in the gastrointestinal tract, omentum, mesentery, or occasionally in the retroperitomenum. They have round to polygonal cells, abundant eosinophilic cytoplasm, and thick-walled blood vessels. Epitheloid leiomyosarcomas demonstrate immunohistochemical negativity for CD 34 and variably positivity for smooth muscle actin. ${ }^{9}$ In contrast to these abovementioned entities, the atypical glomus tumor of uncertain malignant potential exhibits prominent, branching capillary vasculature, perivascular arrangement of tumor cells, uniform cell shape and size, and distinct cell borders. ${ }^{1}$

There are very few instances in the literature of glomus tumors occurring in unusual locations and displaying atypical features. Glomangiosarcomas have been reported in the stomach, lung, and urinary bladder. ${ }^{6,9,10}$ Folpe et al reviewed 22 cases of atypical glomus tumors in various locations, such as finger, ankle, and thigh. ${ }^{1}$ An atypical glomus tumor of the mesentery has been recently reported. ${ }^{11}$ A periurethral mass was found to be a benign glomus tumor. ${ }^{12}$ A search of English literature on PubMed revealed only one reported case of a malignant glomus tumor occurring in the urinary bladder, and there are no previously documented cases of atypical glomus tumors in this organ.

Glomus tumors are generally indolent and are cured by surgical resection, so distinguishing atypical glomus tumors from other entities is valuable. Furthermore, deeply seated or visceral glomus tumors rarely have associated symptoms or symptoms that are referred to the involved organ. ${ }^{3}$ No reported recurrences of atypical glomus tumors after full surgical resection have been reported.

\section{Conclusion}

In our case, the patient has been free of tumor since June 2006. This case of atypical glomus tumor in the urinary bladder exemplifies the range of clinical and histological features of this fascinating lesion. The infrequence of these tumors makes their diagnosis challenging, but careful examination paired with immunohistochemical studies can lead to a correct diagnosis. A noninvasive urothelial carcinoma in collision with an atypical glomus tumor located in the lamina propria should be recognized as separate entities for optimal patient care.

\section{Disclosure}

No conflicts of interest were declared in relation to this paper.

\section{References}

1. Folpe AL, Fanburg-Smith JC, Miettinen M, Weiss SW. Atypical and malignant glomus tumors: analysis of 52 cases, with a proposal for the reclassification of glomus tumors. Am J Surg Pathol. 2001;25:58-64.

2. Goldblum JR Weiss SW, editors. Enzinger and Weiss's Soft Tissue Tumors. 4th ed. 2004; St. Louis: Mosby. pp 751-757.

3. Folpe AL. Pericytic (perivascular) tumours In: Fletcher CDM UK, Mertens F, editors. Pathology and Genetics of Tumours of Soft Tissue and Bone. Lyon, France: IAKC Press, World Health Organization classification of tumours. 2002;5:136-137.

4. Gould EW, Manivel JC, Albores-Saavedra J, Monforte H. Locally infiltrative glomus tumors and glomangiosarcomas. A clinical, ultrastructural, and immunohistochemical study. Cancer. 1990;65:310-318.

5. Folpe AL, Fanburg-Smith JC, Miettinen M, Weiss SW. Atypical and malignant glomus tumors. Am J Surg Pathol. 2001;25:1-12.

6. Gaertner EM, Steinberg DM, Huber M, et al. Pulmonary and mediastinal glomus tumors: report of five cases including a pulmonary glomangiosarcoma: a clinicopathologic study with literature review. Am J Surg Pathol. 2000;24:1105-1114.

7. Yousem S, Hochholzer L. Primary pulmonary hemangiopericytoma. Cancer. 1987;59:549-555.

8. D'Amore ES, Manivel JC, Sung JH. Soft-tissue and meningeal hemangiopericytomas: an immunohistochemical and ultrastructural study. Hum Pathol. 1990;21:414-423.

9. Miettinen M, Paal E, Lasota J, Sobin LH. Gastrointestinal glomus tumors: a clinicopathologic, immunohistochemical, and molecular genetic study of 32 cases. Am J Surg Pathol. 2002;26:301-311.

10. Shim HS, Choi YD, Cho NH. Malignant glomus tumor of the urinary bladder. Arch Pathol Lab Med. 2005;129:940-942.

11. de Bruin AFJ, Verhoef C, den Bakker MA, Geel A. Glomus tumor of the mesentery with atypical features: a case report. Int $J$ Surg Pathol. 2008; 16:440-443.

12. Malowany JI, Rieckenberg RM, Okafo BA, Colgan TJ. Glomus tumor presenting as a periurethral mass. J Low Genit Tract Dis. 2008;12:316.
Pathology and Laboratory Medicine International

\section{Publish your work in this journal}

Pathology and Laboratory Medicine International is a peer-reviewed, open access journal focusing on innovative basic research and translational research related to pathology or human disease. The journal includes original research, updates, case reports, reviews and commentaries on current controversies. The Academic Sponsor

\section{Dovepress}

of this journal is the Chinese American Pathology Association (CAPA). The manuscript management system is completely online and includes a very quick and fair peer-review system. Visit http://www.dovepress.com/testimonials.php to read real quotes from published authors. 